\title{
Light Induced Negative Differential Conductance in Molecular Junctions: Role of Triplet States and Electron-Phonon Interaction
}

\author{
Amir Eskandari-asl ${ }^{\mathrm{a}}$ \\ ${ }^{a}$ Institute of Atomic and Molecular Sciences, Academia Sinica, Taipei 10617, Taiwan
}

\begin{abstract}
In this work we theoretically consider the OPE-3 molecule bridging two metallic leads and show that because of the electron-phonon interaction and the transition of cation to triplet states, we can have a light induced negative differential conductance. Furthermore, we investigate the effects of light intensity and temperature on this phenomena.
\end{abstract}

Keywords: negative differential conductance, triplet states, electron-phonon interaction, light induced transition, master equation

\section{1. introduction}

In the last two decades advances in technology made the realization of molecular junctions and measuring the charge transport through them possible $[1,2,3,4,4,5,6,6,8,9,10]$. At molecular scales the interaction of electrons with the quantum of the vibrational modes, the so called phonons, plays an important role in determining the transport properties. Several novel phenomena including negative differential conductance (NDC), dynamical switching and current hysteresis 11, 12, 13, 14, 15], are believed to originate from electronphonon (e-ph) interaction [16, 17, 18, 19].

One interesting issue which attracted a huge interest recently, is optical control of the electron transport through molecular junctions 20, 21, 22]. Of special interest is to have a light induced current, in which the current can be switched on and off upon illumination. This can be achieved either by exciting the electrons in the case that the incoming light frequency is in resonance with some energy gaps of the molecular states [22, 23, 24, 25, 26], or by using a high intensity coherent quantum light to induce photon side bands that open new transport channels, the so called photon-assisted tunneling [20, 27].

In addition to experiments, the response of molecular junctions to light has been extensively studied in theory. When the lead-molecule coupling is weak,

Email address: amir.eskandari.asl@gmail.com (Amir Eskandari-asl) 
the most efficient approach is to exploit quantum master equations 28, 29, 30]. The effect of the resonant light is included into the master equation by considering a transition rate between ground and excited states, whose magnitude is proportional to the light intensity [31, [32]. In most of theoretical studies the effect of triplet states are ignored due to the small triplet-singlet transition rate [33, 29, 34]. However, it was shown that the triplet states cannot be ignored in the studying the optical response of the molecular junctions, as even though the direct singlet-triplet transition is impossible, one can adjust the parameters in such a way to have the indirect transition through the charged states 31, 32].

In this work we consider the OPE-3 molecule bridging two metallic leads and show that by adjusting the gate voltage one can have the novel phenomena of light induced NDC. This effect is explained using the transitions between the charged and triplet states in the presence of the e-ph interactions and light. It should be noted that in addition to the value for fundamental physics, such behaviors can be very important for engineering future switches and transistors in molecular electronics. In our work however, we predict the additional property of being controllable by light. The rest of this document is organized as follow. In Sec 2 we describe our theoretical method and present the corresponding master equations as well as current formula. In Sec 3 we consider the OPE-3 molecule and using ab initio calculations obtain its phonon mode energies and their corresponding displacements due to electronic state transitions. In order to make the calculations affordable we map nearly degenerate modes into single effective modes, the theory of which is described in the Ref. 35]. We show that by adjusting the gate voltage one can have a light induced NDC and describe the roles of triplet states, e-ph interaction and light to have this phenomena. Moreover, we investigate the effects of light intensity and temperature. Finally, Sec 4 concludes our work.

\section{2. model and method}

Our setup is composed of a molecular junction bridging two leads and capacitively coupled to a gate electrode. The electrons on the molecule are coupled to its vibrational modes which in turn can be dissipated to a thermal bath. We also consider an incoming classical light beam which interacts with the electrons on our molecule. A schematic representation of this setup is shown in Fig1 a. The total Hamiltonian is

$$
\hat{H}=\hat{H}_{\text {mol }}+\hat{H}_{\text {env }}+\hat{H}_{\text {coup }}+\hat{H}_{\text {field }},
$$

where $\hat{H}_{\mathrm{mol}}$ is for the molecule, $\hat{H}_{\mathrm{env}}$ describes the environment involving leads and phonon thermal bath, $\hat{H}_{\text {coup }}$ is responsible for the copuling between the molecular junction and the environment, and $\hat{H}_{\text {field }}$ is the interaction Hamiltonian between the incoming light and the molecule.

The molecular Hamiltonian which involves both electrons and phonons and their interaction, is given by

$$
\hat{H}_{\mathrm{mol}}=\sum_{M} \sum_{r=1}^{R}|M\rangle\langle M|
$$




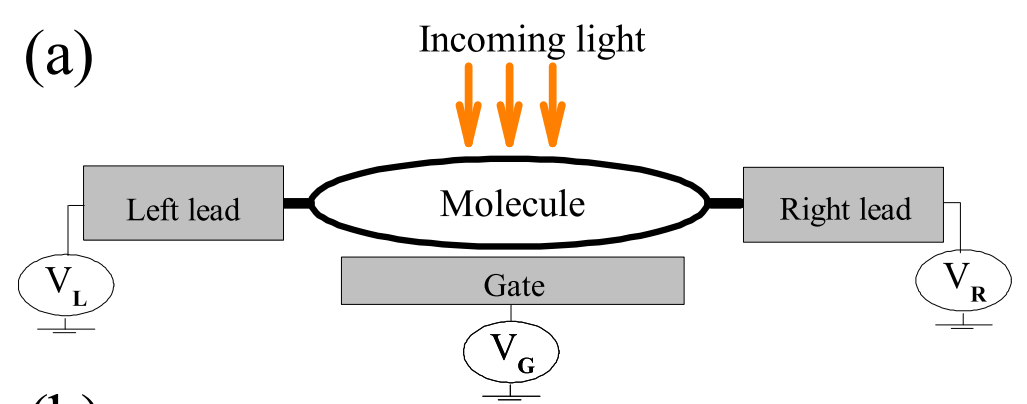

(b)
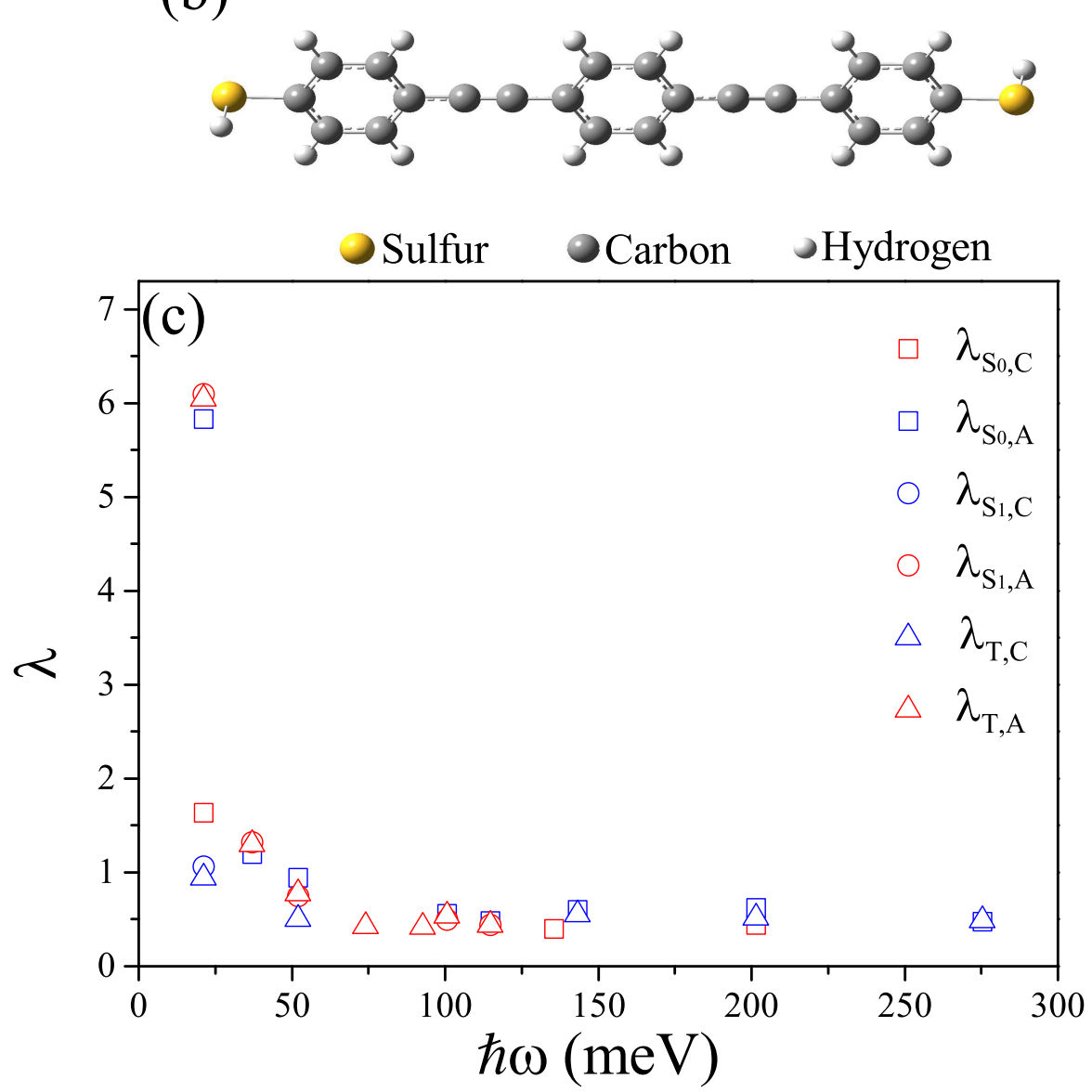

Figure 1: (Color online) (a) Schematic setup. The illuminated molecule is bridging the left and right metallic electrodes while capacitively coupled to the gate electrode. (b) The molecule OPE-3 with the anchoring sulfur atoms. (c) The results of first principle calculations for the electron-phonon coupling strengths. Phonon modes with the energy differences less than $3 \mathrm{meV}$ (equivalent to $35 \mathrm{~K}$ ) are combined and electron-phonon couplings less than 0.4 are ignored. 


$$
\left(\xi_{M}+\hbar \omega_{r}\left[\left(\hat{b}_{r}^{\dagger}-\lambda_{r, M}\right)\left(\hat{b}_{r}-\lambda_{r, M}\right)+1 / 2\right]\right),
$$

where $\{|M\rangle\}$ is the set of many-electron states of the molecule. The electronic energy of the many-electron state $|M\rangle$ is $\xi_{M}=E_{M}-N_{M} e V_{G}$ with $E_{M}$ being the eigenenergy of the electronic Hamiltonian, $N_{M}$ the number of excess electrons in state $|M\rangle$ with respect to the neutral state, and $V_{G}$ the applied gate voltage. $\hat{b}_{r}^{\dagger}\left(\hat{b}_{r}\right)$ denotes the creation (annihilation) operator of the $r$ th phonon mode, while the total number of phonon modes is $R$. Finally, $\lambda_{r, M}$ is the displacement of the $r$ th phonon mode associated with the electronic state $|M\rangle$.

The environment Hamiltonian, $\hat{H}_{\mathrm{env}}=\hat{H}_{\text {leads }}+\hat{H}_{\mathrm{th}}$, is the summation of two parts corresponding to electronic leads and phonon thermal bath, respectively. The leads are considered as non-interacting Fermi gases described by $\hat{H}_{\text {leads }}=\sum_{\alpha=L, R} \varepsilon_{\alpha k \sigma} \hat{c}_{\alpha k \sigma}^{\dagger} \hat{c}_{\alpha k \sigma}$, while the thermal bath is a continuum of quantum harmonic oscillators determined by $\hat{H}_{\text {th }}=\sum_{q} \hbar \omega_{q} \hat{b}_{q}^{\dagger} \hat{b}_{q}$. Here $\hat{c}_{\alpha k \sigma}^{\dagger}$ $\left(\hat{b}_{q}^{\dagger}\right)$ creates an electron (phonon) with energy $\varepsilon_{\alpha k \sigma}\left(\hbar \omega_{q}\right)$ in the lead $\alpha$ (thermal bath). The coupling Hamiltonian is $\hat{H}_{\text {coup }}=\hat{H}_{\mathrm{m}-1}+\hat{H}_{\mathrm{m}-\mathrm{th}}$, where the first term determines the hopping of electrons between the molecule and leads, $\hat{H}_{\mathrm{m}-1}=\sum_{M, M^{\prime}, \alpha k \sigma} V_{\alpha k \sigma, M, M^{\prime}} c_{\alpha k \sigma}^{\dagger}\left|M^{\prime}\right\rangle\langle M| \delta_{N_{M}, N_{M^{\prime}}+1}+$ h.c., while the second term indicates the coupling of the phonons to the thermal bath, $\hat{H}_{\mathrm{m}-\mathrm{th}}=$ $\sum_{q} \sum_{r} t_{q, r}\left(\hat{b}_{q}^{\dagger}+\hat{b}_{q}\right)\left(\hat{b}_{r}^{\dagger}+\hat{b}_{r}\right)$.

For our theoretical investigation we select the following many-electron states of the molecule. The ground and first excited singlet states which are shown, respectively, by $\left|S_{0}\right\rangle$ and $\left|S_{1}\right\rangle$, the triplet states which are denoted by $\left|T^{m}\right\rangle$ in which $m=0, \pm 1$, and the charged anion, $\left|A^{\sigma}\right\rangle$, and cation, $\left|C^{\sigma}\right\rangle$, states in which $\sigma= \pm \frac{1}{2}$. The singlet and triplet states are neutral, i.e., $N_{S_{0}}=N_{S_{1}}=N_{T^{m}}=0$, while for the charged states we have $N_{A^{\sigma}}=-N_{C^{\sigma}}=1$. The electron transport between the molecule and leads changes the molecule from the neutral state to the charged state and vice versa. The spin-orbit coupling could in principle cause transitions between singlet and triplet states with a very low rate which is ignored in our treatment. Finally, the incoming light can interchange the molecular state between $\left|S_{0}\right\rangle$ and $\left|S_{1}\right\rangle$, via the Hamiltonian [36]

$$
\hat{H}_{\text {field }}=-\mathbf{E}(t) \cdot \mathbf{d}_{01}\left|S_{0}\right\rangle\left\langle S_{1}\right|+\text { h.c. },
$$

where $\mathbf{E}$ is the electric field of the incoming light beam and $\mathbf{d}_{01}$ is the dipole moment between the ground and first excited singlet states.

By exploiting a polaron transformation and considering Born-Markov approximation in accordance to the weak molecule-lead coupling (a straight forward generalization of the derivation given in Ref.[32]), the master equation in the Pauli form can be obtained as

$$
\frac{d P_{M \mathbf{n}}}{d t}=\sum_{M^{\prime} \mathbf{n}^{\prime}}\left(k_{M \mathbf{n}, M^{\prime} \mathbf{n}^{\prime}} P_{M^{\prime} \mathbf{n}^{\prime}}-k_{M^{\prime} \mathbf{n}^{\prime}, M \mathbf{n}} P_{M \mathbf{n}}\right),
$$

where $P_{M \mathbf{n}}$ is the probability that the state of molecule be $|M \mathbf{n}\rangle$, in which $\mathbf{n}$ is a $R$-dimensional vector with integer elements $n_{r}$ determining the number 
of phonons in the mode $r$. The transition rate contains contributions from the leads, thermal bath and the electric field of light, and can be written as $k_{M \mathbf{n}, M^{\prime} \mathbf{n}^{\prime}}=\sum_{\alpha=L, R} k_{M \mathbf{n}, M^{\prime} \mathbf{n}^{\prime}}^{\alpha}+k_{M \mathbf{n}, M^{\prime} \mathbf{n}^{\prime}}^{\text {th }}+k_{M \mathbf{n}, M^{\prime} \mathbf{n}^{\prime}}^{\text {field }}$. The first term, which is the transition induced by the lead $\alpha$, is given by

$$
k_{M \mathbf{n}, M^{\prime} \mathbf{n}^{\prime}}^{\alpha}=\Lambda_{M \mathbf{n}, M^{\prime} \mathbf{n}^{\prime}}^{\alpha} \prod_{r=1}^{R} \chi_{n_{r}^{\prime} n_{r}}\left(\lambda_{r, M M^{\prime}}\right)
$$

where

$$
\begin{aligned}
& \Lambda_{M \mathbf{n}, M^{\prime} \mathbf{n}^{\prime}}^{\alpha}=\Gamma^{\alpha} v_{M, M^{\prime}}\left[\delta_{N_{M}, N_{M^{\prime}}+1} f\left(\varepsilon_{M \mathbf{n}, M^{\prime} \mathbf{n}^{\prime}}, \mu_{\alpha}\right)\right. \\
& \left.+\delta_{N_{M}, N_{M^{\prime}}-1}\left(1-f\left(\varepsilon_{M^{\prime} \mathbf{n}^{\prime}, M \mathbf{n}}, \mu_{\alpha}\right)\right)\right]
\end{aligned}
$$

in which $\Gamma^{\alpha}$ is the electron transfer rate between molecular junction and the lead $\alpha$, and $v_{M, M^{\prime}}=\sum_{i}\left|\left\langle M\left|\hat{c}_{i}\right| M^{\prime}\right\rangle\right|^{2}+\sum_{i}\left|\left\langle M\left|\hat{c}_{i}^{\dagger}\right| M^{\prime}\right\rangle\right|^{2}$, where $i$ runs over any complete single-particle basis (including spin) for electrons on the molecule. Moreover, $\delta_{\mu, \nu}$ is the Kronecker delta and $f(\varepsilon, \mu)=1 /\left(1+e^{(\varepsilon-\mu) \beta}\right)$ is the Fermi distribution function with $\beta=1 / k_{B} T$ being the reciprocal temperature. $\varepsilon_{M \mathbf{n}, M^{\prime} \mathbf{n}^{\prime}}=\xi_{M}+\epsilon_{\mathbf{n}}-\xi_{M^{\prime}}-\epsilon_{\mathbf{n}^{\prime}}$, in which $\epsilon_{\mathbf{n}}=\sum_{r=1}^{R} \hbar \omega_{r} n_{r}$, and $\mu_{\alpha}=\mu_{0}+e V_{\alpha}$ is the chemical potential of the lead $\alpha$ (the applied bias voltage is $\left.V=\left(\mu_{L}-\mu_{R}\right) / e=V_{L}-V_{R}\right)$. Moreover, $\lambda_{r, M M^{\prime}}=\left|\lambda_{r, M}-\lambda_{r, M^{\prime}}\right|$, is the dimensionless e-ph coupling of the $r$ th phonon mode. Finally,

$$
\begin{aligned}
& \chi_{n^{\prime} n}(\lambda)=\left|\left\langle n\left|e^{\lambda\left(\hat{b}^{\dagger}-\hat{b}\right)}\right| n^{\prime}\right\rangle\right|^{2}= \\
& e^{-\lambda^{2}}\left|\sum_{j=0}^{n_{<}} \frac{(-1)^{j} \lambda^{2 j+n>-n_{<}} \sqrt{n ! n^{\prime} !}}{j !\left(j+n_{>}-n_{<}\right) !\left(n_{<}-j\right) !}\right|^{2},
\end{aligned}
$$

in which $n_{>(<)}$is the maximum (minimum) of $n$ and $n^{\prime}$.

The coupling to the thermal bath results in thermal excitation-dissipation of phonons with the corresponding rate given by

$$
\begin{aligned}
& k_{M \mathbf{n}, M^{\prime} \mathbf{n}^{\prime}}^{t h}=\delta_{M, M^{\prime}} \gamma^{t h} \sum_{r=1}^{R}\left[\delta_{n_{r}, n_{r}^{\prime}+1}\left(n_{r}^{\prime}+1\right) n_{t h}\left(\omega_{r}\right)\right. \\
& \left.+\delta_{n_{r}, n_{r}^{\prime}-1} n_{r}^{\prime}\left(n_{t h}\left(\omega_{r}\right)+1\right)\right]
\end{aligned}
$$

where $\gamma^{\text {th }}$ determines the relaxation rate of phonons to the thermal bath and $n_{t h}\left(\omega_{r}\right)=1 /\left(e^{\beta \hbar \omega_{r}}-1\right)$.

The incoming light can only cause a transition between the states $\left|S_{0}\right\rangle$ and $\left\langle S_{1}\right|$ with the rate given by

$$
k_{S_{0} \mathbf{n}, S_{1} \mathbf{n}^{\prime}}^{\mathrm{field}}=k_{S_{1} \mathbf{n}, S_{0} \mathbf{n}^{\prime}}^{\mathrm{field}}=k^{\mathrm{field}} \prod_{r=1}^{R} \chi_{n_{r}^{\prime} n_{r}}\left(\lambda_{r, M M^{\prime}}\right),
$$


where the instantaneous emission rate is neglected compared to $k^{\text {field }}$, which is the optical induced transition rate tunable with the light intensity [32].

In this work we consider the phonons to be strongly coupled to the thermal bath so that $\gamma^{t h}$ is much larger than the transition rates induced by light and leads. In such a case phonon population is instantly equilibrated so that $P_{M, \mathbf{n}}=$ $e^{-\beta \epsilon_{\mathbf{n}}} P_{M, \mathbf{0}}$. As the phonon distribution is now thermal, the total population of electronic states, $P_{M}=\sum_{\mathbf{n}} P_{M, \mathbf{n}}$ should be investigated rather than each individual $P_{M, \mathbf{n}}$. It's straightforward to show that the dynamics of these equili-

brated probability distributions is given as $d P_{M} / d t=\sum_{M^{\prime}}\left(k_{M, M^{\prime}} P_{M^{\prime}}-k_{M^{\prime}, M} P_{M}\right)$, where $k_{M, M^{\prime}}=\sum_{\alpha=L, R} k_{M, M^{\prime}}^{\alpha}+k_{M, M^{\prime}}^{\text {field }}$, where

$$
k_{M, M^{\prime}}^{\alpha}=\frac{1}{Z} \sum_{\mathbf{n}, \mathbf{n}^{\prime}} \Lambda_{M \mathbf{n}, M^{\prime} \mathbf{n}^{\prime}}^{\alpha} \prod_{r=1}^{R} \chi_{n_{r}^{\prime} n_{r}}\left(\lambda_{r, M M^{\prime}}\right) e^{-\beta \epsilon_{\mathbf{n}^{\prime}}}
$$

in which

$$
Z=\sum_{\mathbf{n}} e^{-\beta \epsilon_{\mathbf{n}}}=\prod_{r=1}^{R} \frac{1}{1-e^{-\beta \hbar \omega_{r}}}
$$

and

$$
k_{M, M^{\prime}}^{\text {field }}=k^{\text {field }}\left(\delta_{M, S_{0}} \delta_{M^{\prime}, S_{1}}+\delta_{M, S_{1}} \delta_{M^{\prime}, S_{0}}\right) .
$$

Investigating the electron population change, one can show that the electrical current from lead $\alpha$ to the molecule is

$$
I^{\alpha}=e \sum_{M, M^{\prime}} P_{M} k_{M^{\prime}, M}^{\alpha}\left(\delta_{N_{M}+1, N_{M^{\prime}}}-\delta_{N_{M}-1, N_{M^{\prime}}}\right) .
$$

\section{Results and discussions}

We consider the OPE-3 molecule with the structure as shown in Fig[1 b. Using the Gaussian 09 software at the B3LYP 37] level and exploiting the cc-pVDZ basis set, the geometry optimization and phonon mode energies are calculated. Moreover, performing the Duschinsky transformation 38] using the DUSHIN 39] code, we computed the e-ph couplings for all possible charge transitions in the truncated Fock space of molecular electronic states. For a thermal bath at the temperature of $35 \mathrm{~K}$, the phonon modes with the energy differences less than $3 \mathrm{meV}$ can be considered as nearly-degenerate. For the transport calculations, nearly-degenerate modes can be mapped into an effective mode with the effective Huang-Rhys factor (which is the square of e-ph coupling strength) being the average of the Huang-Rhys factors of the original modes [35]. The effective resulting e-ph couplings are shown in Fig. 1 c, where the effective couplings less than 0.4 are ignored.

The electronic state energies are obtained to be $E_{S_{0}}=0, E_{S_{1}}=3.029$ $\mathrm{eV}, E_{T}=1.871 \mathrm{eV}, E_{C}=6.178 \mathrm{eV}$ and $E_{A}=-1.365 \mathrm{eV}$, in which for the 


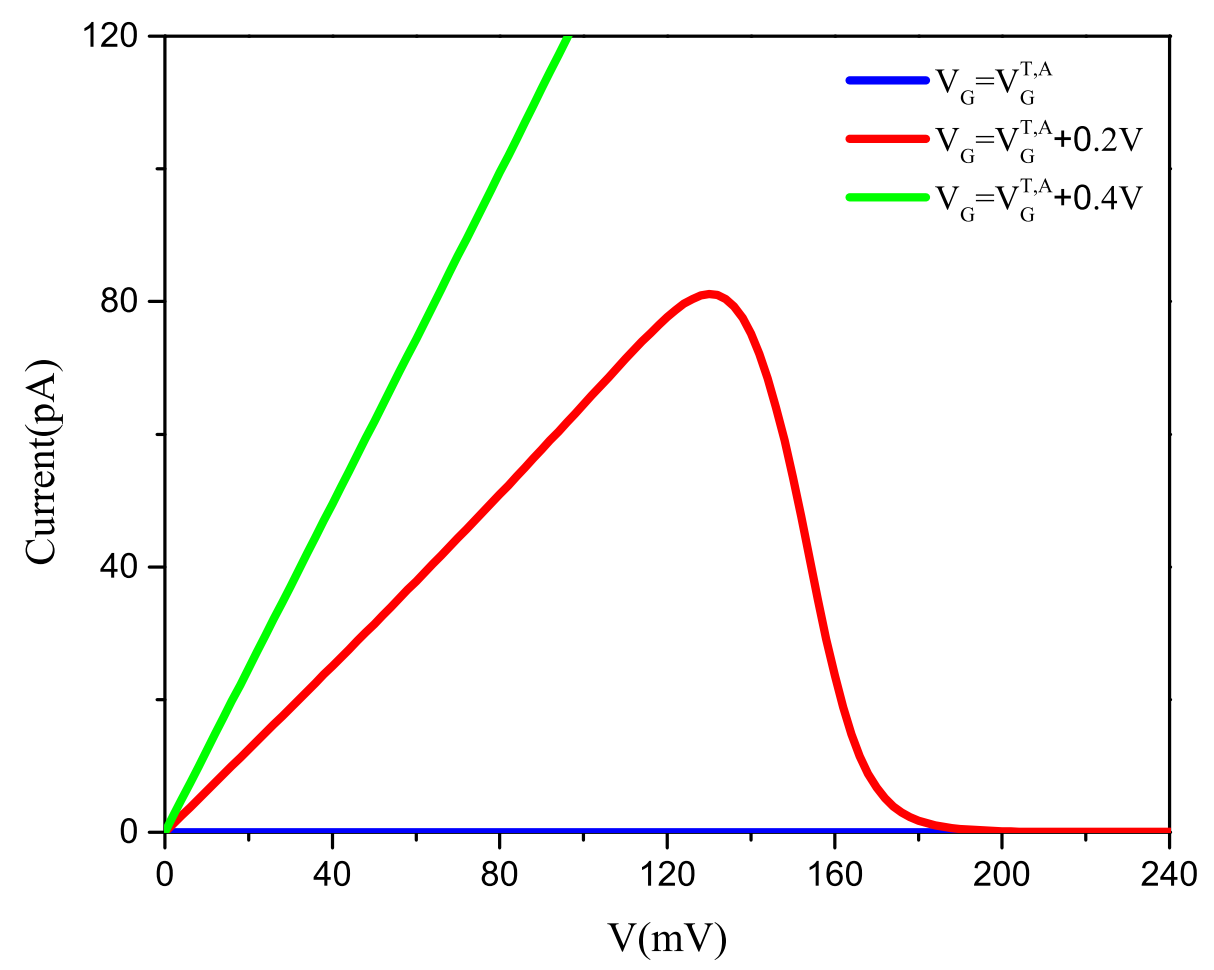

Figure 2: (Color online) IV curves for several values of the gate voltages. In order to have NDC we have to adjust the gate voltage to be slightly more than $V_{G}^{T, A}$, as in the red curve here. The light induced transition rate is $k^{\text {field }}=10^{12} \mathrm{~s}^{-1}$ and the temperature is $35 \mathrm{~K}$, which corresponds to $k_{B} T=3 \mathrm{meV}$.

anion and cation states an image charge correction of $-0.3 \mathrm{eV}$ is considered 32]. Moreover, the non-biased chemical potential of the metallic leads is assumed to be $\mu_{0}=-5.3 \mathrm{eV}$. The charge transport through molecule can be controlled by applying a gate voltage. The gate voltage that aligns triplet states with anion states is $V_{G}^{T, A}=\left(E_{A}-E_{T}-\mu_{0}\right) / e=2.064 \mathrm{~V}$ (the concept of state alignment is described in the Refs. 31, 32 ) and in our investigations other gate voltages are measured with respect to $V_{G}^{T, A}$. In our calculations we consider a symmetric lead-molecule coupling with the rate $\Gamma=\Gamma^{R}=\Gamma^{L}=0.1 \mathrm{meV}$, and the bias voltage is applied symmetrically, $V_{L}=-V_{R}=V / 2$.

In Fig. 2 we considered three values of the gate voltage and obtained the corresponding IV curves. The light induced transition rate is $k^{\text {field }}=10^{12} \mathrm{~s}^{-1}$ and the temperature is $35 \mathrm{~K}$, which corresponds to $k_{B} T=3 \mathrm{meV}$. It's seen that for the gate voltage $V_{G}=V_{G}^{T, A}$ we have no current at all. In order to understand this behavior, we have to investigate the state transition rates. At this gate voltage the important transitions are $S_{0} \leftrightarrows S_{1} \rightarrow C \rightarrow T$, as a result of which the molecule is totally populated with the triplet states and no steady-state 
current can be established. In order to have an electrical current in this case, one has to increase the bias voltage to overcome the Franck-Condon blockade and make the transition $T \rightarrow A$ possible (not shown in the figure).

By increasing the gate voltage, as it is the case for $V_{G}=V_{G}^{T, A}+0.2 \mathrm{~V}$ and $V_{G}=V_{G}^{T, A}+0.4 \mathrm{~V}$ in Fig. 2, the transition $S_{1} \rightarrow C$ is blocked for low bias voltages and the cation state population vanishes, which in turn makes the triplet states unreachable, as only the cation states can make transition to them in this regime. Consequently, it is possible to have a low bias current of the order of $10-100 \mathrm{pA}$ which is measurable in experimental setups [5]. The steady-state current is now maintained by the transitions $S_{0} \leftrightarrows S_{1} \rightarrow A \rightarrow S_{0}$. One should notice that because of the Franck-Condon blockade resulting from e-ph coupling, the transition rates in and out of the anion states are different depending on the lead which induces them. In short, $K_{A, S_{1}}^{L} \neq K_{A, S_{1}}^{R}$ and $K_{S_{0}, A}^{L} \neq K_{S_{0}, A}^{R}$, which is the reason of having current and determines the important role of phonons.

For the gate voltage $V_{G}=V_{G}^{T, A}+0.2 \mathrm{~V}$ ( the red curve in Fig. 2), we have NDC as the current is decreased by increasing the bias voltage above $V \simeq 130 \mathrm{mV}$. This behavior is explained by noticing that at higher bias voltages a transition from the state $S_{1}$ to the cation states gradually turns on, which in turn decay to the triplet states and make them populated. Because of the Franck-Condon blockade, the triplet states make no transitions to the charged states which means electrons cannot jump into or outside of the molecule. Consequently, the current is blocked and NDC emerges. It is clear from this discussion that in order to have NDC we need both e-ph coupling and the presence of triplet states. Moreover, it is noteworthy that this phenomenon occurs at low bias voltages, hence the phonon modes with low energies play the most important role.

In addition to e-ph coupling, the interaction with the incoming classical light is crucial for having NDC. This is explicitly shown in Fig 3, where at the fixed gate voltage of $V_{G}=V_{G}^{T, A}+0.2$ and temperature of $35 \mathrm{~K}$, we considered several values of the light induced transitions, $k^{\text {field }}$. Reducing the light intensity reduces the transition rates of the $S_{0} \leftrightarrows S_{1}$ processes, therefore, the probability that the molecule stays in the neutral state $S_{0}$ and maintains no electrical current increases. On the other hand, by increasing the light intensity the peak value of current reaches its saturated value, as other transitions (i.e., $S_{1} \rightarrow$ $A \rightarrow S_{0}$ ) are not affected. Moreover, according to our former discussions the bias voltage at which the NDC starts is not determined by the $S_{0} \leftrightarrows S_{1}$ processes which makes it independent of the light intensity.

In order to investigate the effect of temperature in NDC, in Fig. 4 we show the IV curves for three different temperatures with the fixed gate voltage of $V_{G}=V_{G}^{T, A}+0.2 \mathrm{~V}$ and the light induced transition rate of $k^{\text {field }}=10^{12} \mathrm{~s}^{-1}$. It should be noted that our results for the case $\mathrm{T}=11.5 \mathrm{~K}$ are rough, since we have done the effective combination for the temperatures more than or equal to $35 \mathrm{~K}$. Our results indicate that by decreasing the temperature the bias voltage at which we have NDC is increased, as well as the peak value of current. As we already explained, the NDC starts at the bias voltage where the $S_{1} \rightarrow$ 


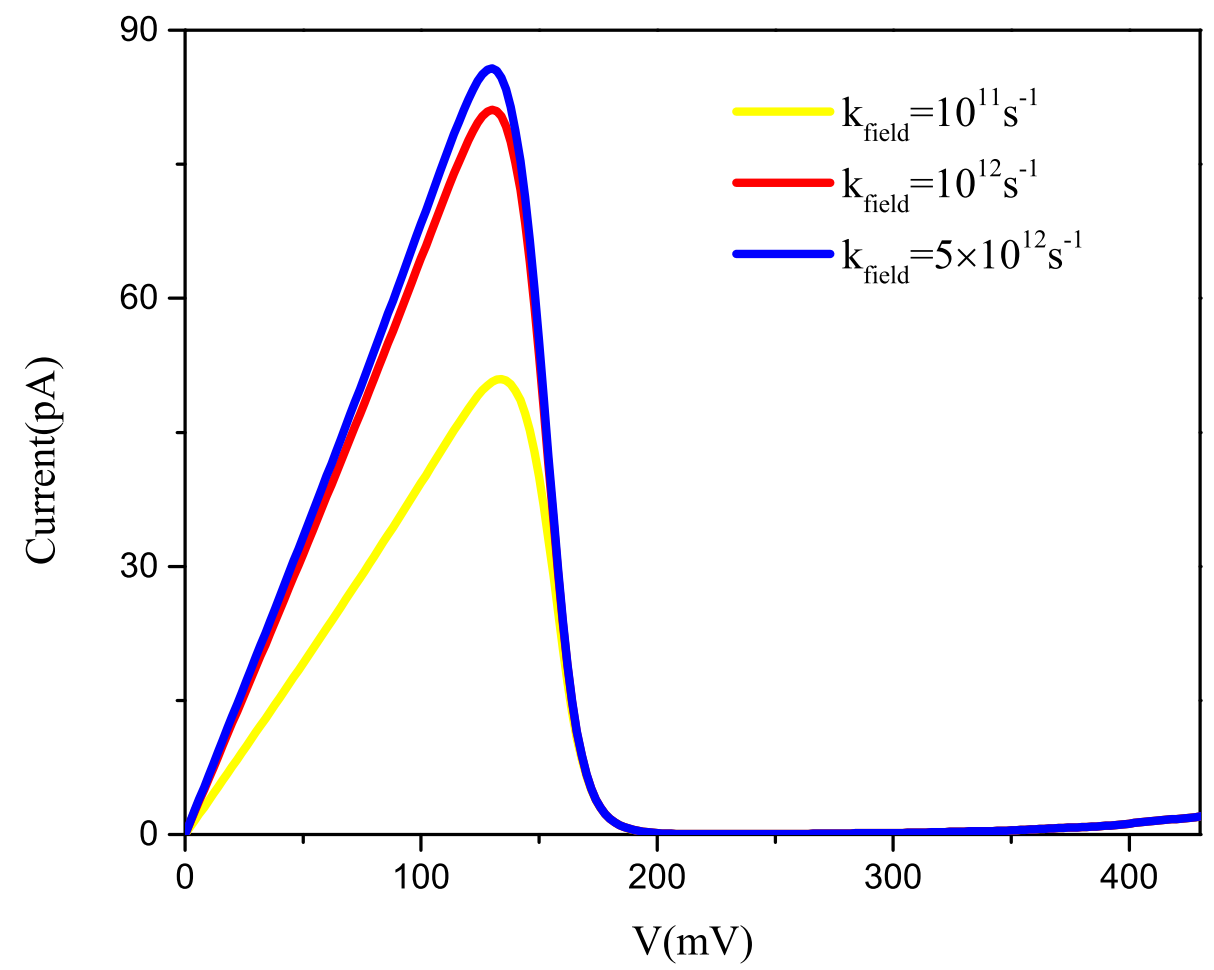

Figure 3: (Color online) IV curves for several values of the light induced transition rates. Reducing the light intensity reduces $k^{\text {field }}$ which lowers the peak in IV curve which means weakening the NDC. The gate voltage is $V_{G}^{T, A}+0.2 \mathrm{~V}$ and the temperature is $35 \mathrm{~K}$. 


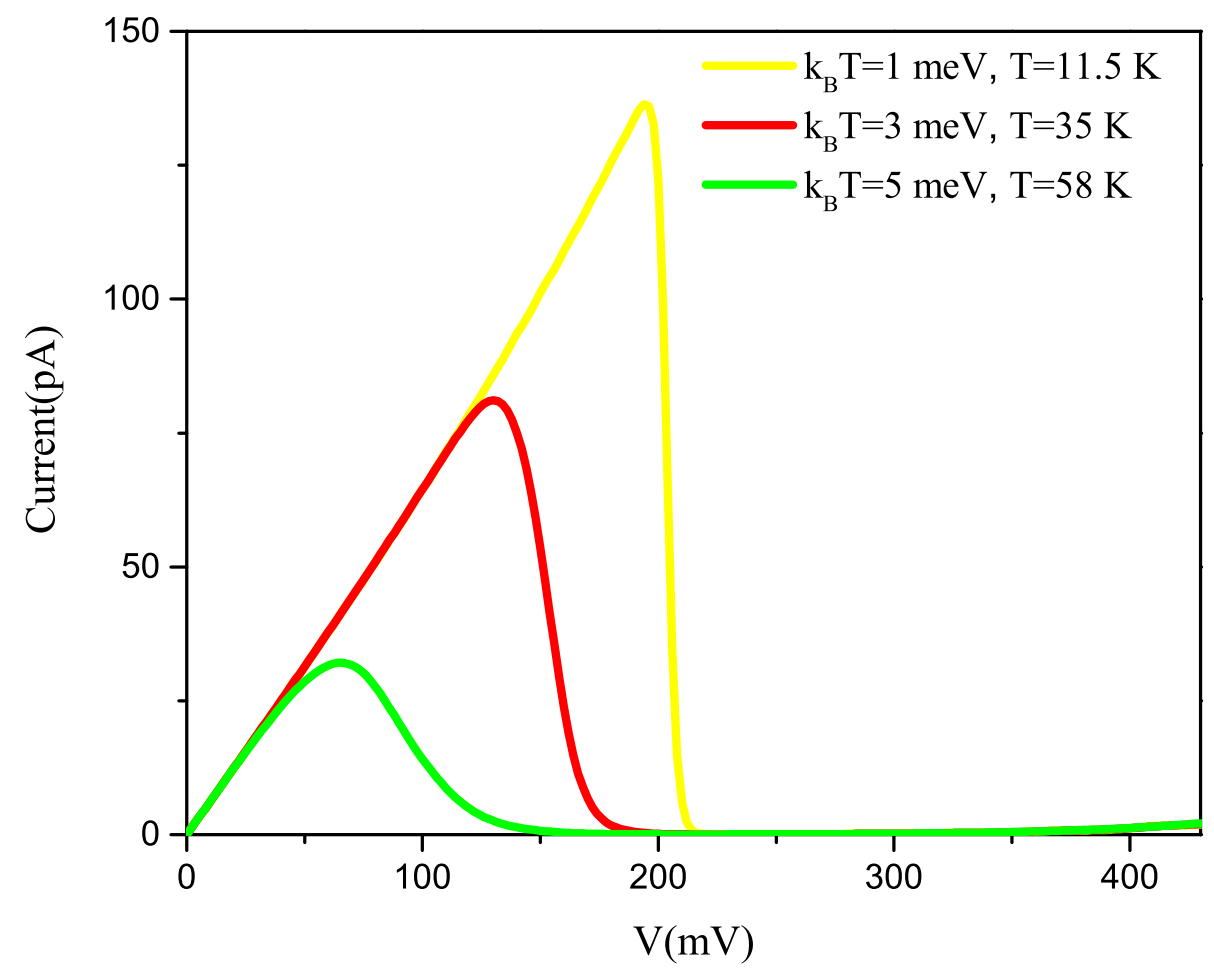

Figure 4: (Color online) IV curves for several values of temperatures. Lowering the temperature strengthens the NDC, while it still persists by increasing temperature. The gate voltage is $V_{G}^{T, A}+0.2 \mathrm{~V}$ and the light induced transition rate is $k^{\text {field }}=10^{12} \mathrm{~s}^{-1}$. 
$C$ transition becomes possible. At zero temperature the Fermi distribution function is a step function and this bias voltage (which is the peak voltage in the IV curve) can be obtained by demanding $K_{C, S_{1}}^{R}=0$ which results in $V=2\left(E_{C}-E_{S_{1}}+e V_{G}+\mu_{0}\right) / e=226 \mathrm{mV}$. By increasing the temperature the step in the Fermi function gets smooth, which makes the $S_{1} \rightarrow C$ transition possible at lower bias voltages and decreases the peak value of current.

\section{Conclusions}

In conclusion, we studied the OPE-3 molecular junction and showed that the transition to triplet states, e-ph interaction and light illumination can result in the novel phenomena of light induced NDC. By adjusting the gate voltage, one can see that there is a bias voltage after which a transition from the excited singlet states to the cation states is possible, which in turn decay to the triplet states and block the light induced electronic current. Consequently, we have NDC. We showed that by reducing the light intensity the current peak is decreased while the bias voltage at which NDC starts is fixed. On the other hand, increasing the light intensity we approach the saturated current and NDC values. By increasing the temperature the step functions of the Fermi distribution functions of the leads get smooth, which make the transition to the cation state and consequently the NDC possible at lower temperatures. Our results can be of potential importance for constructing future controllable molecular switches and transistors.

\section{ACKNOWLEDGMENT}

We acknowledge useful discussions with Liang-Yan Hsu, Bo Fu, and QianRui Huang.

\section{References}

[1] D. Strachan, D. Smith, D. Johnston, T.-H. Park, M. J. Therien, D. Bonnell, A. Johnson, Controlled fabrication of nanogaps in ambient environment for molecular electronics, Applied Physics Letters 86 (2005) 043109.

[2] X. Cui, A. Primak, X. Zarate, J. Tomfohr, O. Sankey, A. L. Moore, T. A. Moore, D. Gust, G. Harris, S. Lindsay, Reproducible measurement of single-molecule conductivity, science 294 (2001) 571-574.

[3] J. Park, A. N. Pasupathy, J. I. Goldsmith, C. Chang, Y. Yaish, J. R. Petta, M. Rinkoski, J. P. Sethna, H. D. Abruña, P. L. McEuen, et al., Coulomb blockade and the kondo effect in single-atom transistors, Nature 417 (2002) $722-725$.

[4] B. Xu, N. J. Tao, Measurement of single-molecule resistance by repeated formation of molecular junctions, science 301 (2003) 1221-1223. 
[5] S. Kubatkin, A. Danilov, M. Hjort, J. Cornil, J.-L. Bredas, N. StuhrHansen, P. Hedegård, T. Bjørnholm, Single-electron transistor of a single organic molecule with access to several redox states, Nature 425 (2003) 698-701.

[6] T. Dadosh, Y. Gordin, R. Krahne, I. Khivrich, D. Mahalu, V. Frydman, J. Sperling, A. Yacoby, I. Bar-Joseph, Measurement of the conductance of single conjugated molecules, Nature 436 (2005) 677-680.

[7] L. Venkataraman, J. E. Klare, C. Nuckolls, M. S. Hybertsen, M. L. Steigerwald, Dependence of single-molecule junction conductance on molecular conformation, Nature 442 (2006) 904-907.

[8] K. Moth-Poulsen, T. Bjørnholm, Molecular electronics with single molecules in solid-state devices, Nature nanotechnology 4 (2009) 551.

[9] N. Xin, J. Guan, C. Zhou, X. Chen, C. Gu, Y. Li, M. A. Ratner, A. Nitzan, J. F. Stoddart, X. Guo, Concepts in the design and engineering of singlemolecule electronic devices, Nature Reviews Physics 1 (2019) 211-230.

[10] A. Morteza Najarian, A. Bayat, R. L. McCreery, Orbital control of photocurrents in large area all-carbon molecular junctions, Journal of the American Chemical Society 140 (2018) 1900-1909.

[11] B. Xu, Y. Dubi, Negative differential conductance in molecular junctions: an overview of experiment and theory, Journal of Physics: Condensed Matter 27 (2015) 263202.

[12] C. Li, D. Zhang, X. Liu, S. Han, T. Tang, C. Zhou, W. Fan, J. Koehne, J. Han, M. Meyyappan, A. M. Rawlett, D. W. Price, J. M. Tour, Applied Physics Letters 82 (2003) 645.

[13] E. Lortscher, J. Ciszek, J. Tour, H. Riel, Small 2 (2006) 973.

[14] P. Liljeroth, J. Repp, G. Meyer, Sience 317 (2007) 1203.

[15] J. D. Le, Y. He, T. R. Hoye, C. C. Mead, R. A. Kiehl, Negative differential resistance in a bilayer molecular junction, Applied physics letters 83 (2003) $5518-5520$.

[16] M. Galperin, M. A. Ratner, A. Nitzan, Hysteresis, switching, and negative differential resistance in molecular junctions: a polaron model, Nano letters 5 (2005) 125-130.

[17] M. Galperin, A. Nitzan, M. A. Ratner, The non-linear response of molecular junctions: the polaron model revisited, Journal of Physics: Condensed Matter 20 (2008) 374107.

[18] A. Eskandari-asl, Bi-stability in single impurity anderson model with strong electron-phonon interaction (polaron regime), Physica B: Condensed Matter 497 (2016) 11-13. 
[19] A. Eskandari-asl, Interplay of electron-electron and electron-phonon interactions in molecular junctions, Physics Letters A 383 (2019) 2120-2123.

[20] K. Yoshida, K. Shibata, K. Hirakawa, Terahertz field enhancement and photon-assisted tunneling in single-molecule transistors, Physical Review Letters 115 (2015) 138302.

[21] C. Jia, A. Migliore, N. Xin, S. Huang, J. Wang, Q. Yang, S. Wang, H. Chen, D. Wang, B. Feng, et al., Covalently bonded single-molecule junctions with stable and reversible photoswitched conductivity, Science 352 (2016) 14431445 .

[22] J. Zhou, K. Wang, B. Xu, Y. Dubi, Photoconductance from exciton binding in molecular junctions, Journal of the American Chemical Society 140 (2018) $70-73$.

[23] E. Petrov, V. Leonov, V. Snitsarev, Transient photocurrent in molecular junctions: Singlet switching on and triplet blocking, The Journal of Chemical Physics 138 (2013) 184709.

[24] M. Kornbluth, A. Nitzan, T. Seideman, Light-induced electronic nonequilibrium in plasmonic particles, The Journal of chemical physics 138 (2013) 174707.

[25] B. Fainberg, T. Seideman, Photoinduced current in molecular conduction junctions with semiconductor contacts, physica status solidi (a) 209 (2012) 2433-2436.

[26] S. Battacharyya, A. Kibel, G. Kodis, P. A. Liddell, M. Gervaldo, D. Gust, S. Lindsay, Optical modulation of molecular conductance, Nano letters 11 (2011) 2709-2714.

[27] E.-D. Fung, O. Adak, G. Lovat, D. Scarabelli, L. Venkataraman, Too hot for photon-assisted transport: hot-electrons dominate conductance enhancement in illuminated single-molecule junctions, Nano letters 17 (2017) $1255-1261$.

[28] V. May, O. Kühn, Optical field control of charge transmission through a molecular wire. i. generalized master equation description, Physical Review B 77 (2008) 115439.

[29] V. May, O. Kuehn, Optical field control of charge transmission through a molecular wire. ii. photoinduced removal of the franck-condon blockade, Physical Review B 77 (2008) 115440.

[30] B. Muralidharan, A. Ghosh, S. Datta, Probing electronic excitations in molecular conduction, Physical Review B 73 (2006) 155410. 
[31] B. Fu, M. A. Mosquera, G. C. Schatz, M. A. Ratner, L.-Y. Hsu, Photoinduced anomalous coulomb blockade and the role of triplet states in electron transport through an irradiated molecular transistor, Nano letters 18 (2018) 5015-5023.

[32] B. Fu, L.-Y. Hsu, Photoinduced anomalous coulomb blockade and the role of triplet states in electron transport through an irradiated molecular transistor. ii. effects of electron-phonon coupling and vibrational relaxation, The Journal of Chemical Physics 151 (2019) 054704.

[33] L. Wang, V. May, Laser pulse induced transient currents through a single molecule, Physical Chemistry Chemical Physics 13 (2011) 8755-8768.

[34] L. Wang, V. May, Charge transmission through single molecules: Effects of nonequilibrium molecular vibrations and photoinduced transitions, Chemical Physics 375 (2010) 252-264.

[35] A. Eskandari-asl, Role of nearly-degenerate vibrational modes in electron transport through a molecular junction, 2020.

[36] G. C. Schatz, M. A. Ratner, Quantum mechanics in chemistry, Courier Corporation, 2002.

[37] A. D. Becke, Becke's three parameter hybrid method using the lyp correlation functional, The Journal of Chemical Physics 98 (1993) 5648-5652.

[38] F. Duschinsky, The importance of the electron spectrum in multi atomic molecules. concerning the franck-condon principle, Acta physicochimica URSS 7 (1937) 551-566.

[39] J. R. Reimers, A practical method for the use of curvilinear coordinates in calculations of normal-mode-projected displacements and duschinsky rotation matrices for large molecules, The Journal of Chemical Physics 115 (2001) 9103-9109. 